\title{
Knochenentnahme am Becken aus Sicht der Mund-, Kiefer- und Gesichtschirurgie
}

Nora Lautner, Frank Hölzle, Ali Modabber

Autologe Transplantate zählen aufgrund ihrer günstigen Eigenschaften zum „Goldstandard“ des Knochenaufbaus in der Mund-, Kiefer- und Gesichtschirurgie, da sich die Kombination aus Osteoinduktion, Osteokonduktion und Osteogenese positiv auf das Langzeitergebnis auswirkt. Durch das histokompatible Knochentransplantat desselben Individuums werden Immunreaktionen vermieden und die Übertragung von Infektionserkrankungen verhindert.

Bedingt durch die Geschehnisse der beiden Weltkriege entstand der vermehrte Bedarf von Mittelgesichtsrekonstruktionen durch knöcherne Transplantate [1, 2]. Schnell zeigten sich die Vorzüge von Beckenkammtransplantaten, da sie eine schnelle Einheilungszeit sowie eine hohe Toleranz gegenüber Infektionen aufwiesen. Zum Großteil erfolgte schon zur damaligen Zeit die Entnahme des benötigten Knochens im anterioren Becken [3]. Jedoch stellte schon damals der posteriore Beckenkamm eine interessante Alternative dar, wenn zur Rekonstruktion großer Defekte geplant wurde.

Die Knochenentnahme aus dem Becken ist im Vergleich zu anderen Entnahmestellen, wie Tibia, distaler Femur, Fibula oder Rippen, weit verbreitet [4]. Entsprechend dem augmentativen Bedarf kann - je nach Entnahmestelle und Transplantatart (frei oder mikrovaskulär) - die genaue Menge und erwünsche Qualität des zu transplantierenden Knochens (Kortikalis, kortikospongiöser Knochen, Spongiosa) für die zu rekonstruierende Region präzise am Patienten ausgewählt werden.

\section{Freies Beckenkammtransplantat}

Ein freies Beckenkammtransplantat eignet sich sehr gut, um z. B. eine entstandene Knochenhöhle nach einer Zystektomie im Kiefer zu versorgen. Auch bei sekundären Rekonstruktionen zum Ausgleich von Impressionen und Konturunregelmäßigkeiten nach Frakturen wird die Modellierbarkeit und Formbarkeit eines freien Knochentransplantats eingesetzt. Freier Beckenkamm kann sowohl zur sekundären Osteoplastik bei Kieferspalten verwendet werden als auch zu Auflagerungsosteoplastiken im Ober- und Unterkiefer, um eine geeignete knöcherne Basis vor geplanter Implantation vorzubereiten. Des Weiteren ermöglicht ein freies Transplantat die Erhöhung des Tuberculum articulare, im Sinne einer Auflagerungs- osteoplastik nach Rehrmann, wodurch die Bewegung des Kondylus nach anterior eingeschränkt wird. Autologer Knochen wird auch zum Ersatz des Kondylus bei Aplasie oder Hypoplasie geschätzt, da damit prinzipiell die Chance einer Wachstumsinduktion gegeben ist, wodurch evtl. weitere korrigierende Operationen in ihrem Ausmaß reduziert werden oder sich sogar erübrigen können [5-7]. Vor allem im Kindesalter wird Beckenknochen mit seiner Kambiumschicht dem Rippentransplantat bis zum Erreichen des 8. Lebensjahres vorgezogen, da er in diesem Alter stabiler als ein Rippentransplantat und damit leichter am aufsteigenden Ast zu befestigen ist [8].

\section{Freie Knochenentnahme aus dem anterioren Becken}

Die Knochenentnahme aus dem anterioren Becken wird aufgrund ihrer Vorteile und ihrer großen Variationsbreite häufig für eine geplante Augmentation im mund-, kieferund gesichtschirurgischen Bereich gewählt. Zu den Vorteilen dieser Knochenentnahme zählen das ausreichende Knochenangebot dieser Region, die gute Verfügbarkeit und die Möglichkeit des zeitgleichen Arbeitens mit 2 Teams, wodurch die Operationszeit deutlich verkürzt werden kann [9-14]. Es existieren diverse Arten der Knochenqualität (kortikal, kortikospongiös, rein spongiös; monokortikal/bikortikal) sowie unterschiedlichste Zugänge (Trepanation [15, 16], lateraler Zugang [17, 18], superiorer Zugang [19], medialer Zugang [17, 20-22]). Als nachteilhaft gelten jedoch eine erhöhte Infektionsrate, die mögliche Frakturgefahr des Beckens, sensorische oder funktionelle Defizite, die Ausbildung von Hämatomen sowie im Vergleich zur posterioren Beckenknochenentnahme die deutlich stärker ausgebildete Narbenbildung $[4,12,23]$ und das geringere Knochenangebot [11].

In der Regel erfolgt nach der Positionierung des Patienten in Rückenlage die Inzision ca. $2 \mathrm{~cm}$ posterior der Spina iliaca anterior superior auf der Crista iliaca ( $\vee$ Abb. 1). Bei geplanter Transplantation eines großen Knochensegments wird aus biomechanischen Gründen ein Abstand von $3 \mathrm{~cm}$ zur Spina iliaca anterior superior angeraten [24]. Die Entnahme wird durch eine gezielte Zuhilfenahme von Tüchern oder Sandsäcken unter dem Becken erleichtert. Durch die angeführte Schnittführung kann einerseits eine spätere Fraktur im Bereich der Becken- 


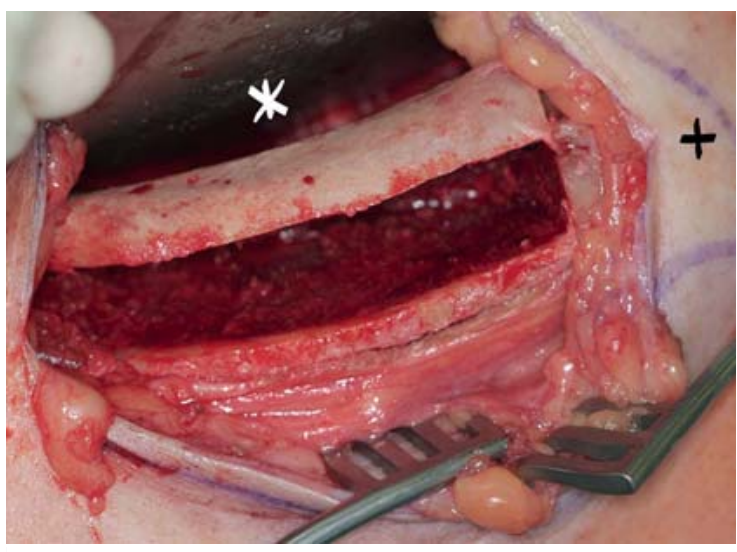

- Abb. 1 Freies Beckenkammtransplantat nach der Osteotomie im anterioren Becken ( ${ }^{*}$ medial, + anterior).

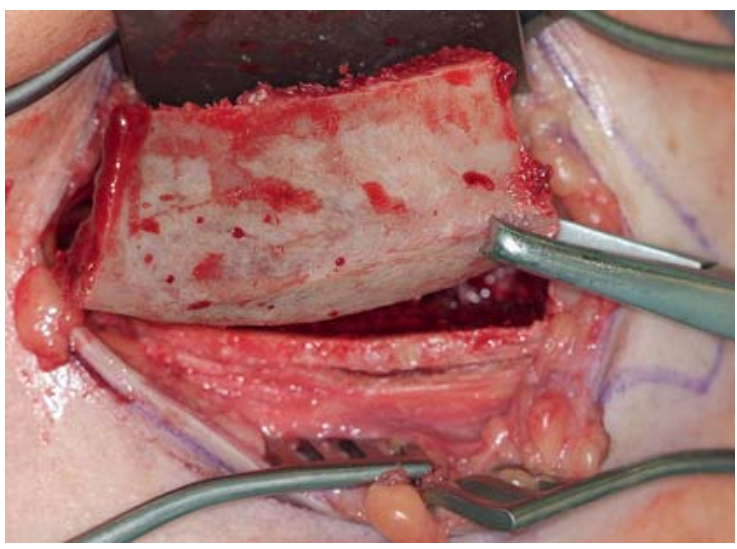

- Abb. 2 Entnahme des freien kortikospongiösen Beckenkammtransplantats aus dem anterioren Becken und Belassen der äußeren Kortikalis.

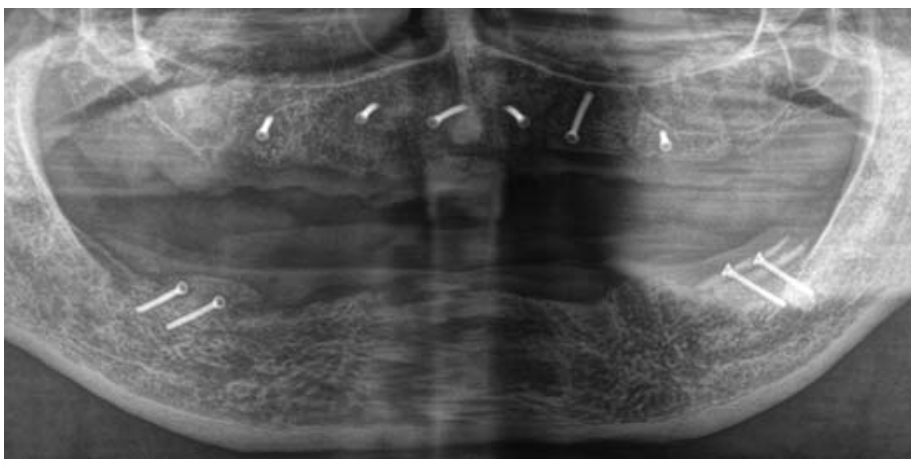

- Abb. 3 Postoperatives Orthopantomogramm (OPG) nach dem Einbringen des freien Beckenkammknochens am Ober- und Unterkiefer zur Augmentation und Fixation mit Osteosyntheseschrauben.

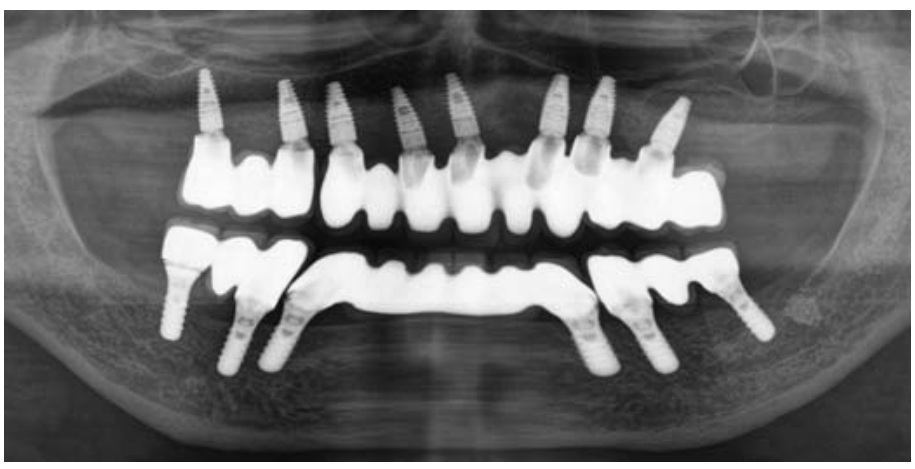

- Abb. 4 OPG nach implantatprothetischer Versorgung im Ober- und Unterkiefer. schaufel vermieden und andererseits eine Verletzung des N. cutaneus femoralis lateralis, M. sartorius oder Lig. inguinale verhindert werden [11,25]. Eine unvorsichtige Penetration der inneren Kortikalis während der Knochenentnahme kann zu einer Verletzung der neurovaskulären Strukturen in der Fossa iliaca führen - dazu zählen z. B. der N. ilioinguinalis, N. cutaneus femoralis lateralis oder auch N. femoralis [25]. Durch eine vorsichtige Retraktion der abdominellen Wand und des M. iliacus können jedoch Verletzungen vermieden werden.

\section{Freie Knochenentnahme aus dem posterioren Becken}

Die Knochenentnahme aus der posterioren Beckenregion zählt zu den häufigen Eingriffen in der Orthopädie und wird vielfach in der Wirbelsäulenchirurgie zum Einsatz gebracht.

In der Mund-, Kiefer- und Gesichtschirurgie kann diese Methode herangezogen werden, wenn große Mengen an Knochen zur Rekonstruktion benötigt werden [10,22, 26 -28]. Dingmann beschreibt im Jahr 1950 als einer der ersten Autoren die Verwendung von freiem Beckenkamm zur Behandlung von fazialen oder kranialen Defekten [29]. Nachteilig ist allerdings die erforderliche Umlagerung des Patienten, was eine signifikante Verlängerung der OP-Zeit mit sich bringt $[10,13,28,30]$.

Zu den bekannten Nachteilen und potenziellen Komplikationen der posterioren Entnahme zählen die im Zusammenhang mit der intraoperativen Patientenumpositionierung erforderliche Bauchlage, die Verletzung des Iliosakralgelenks durch eine übermäßige Ablösung der ligamentären Strukturen [25], Verletzungen des N. ischia- 

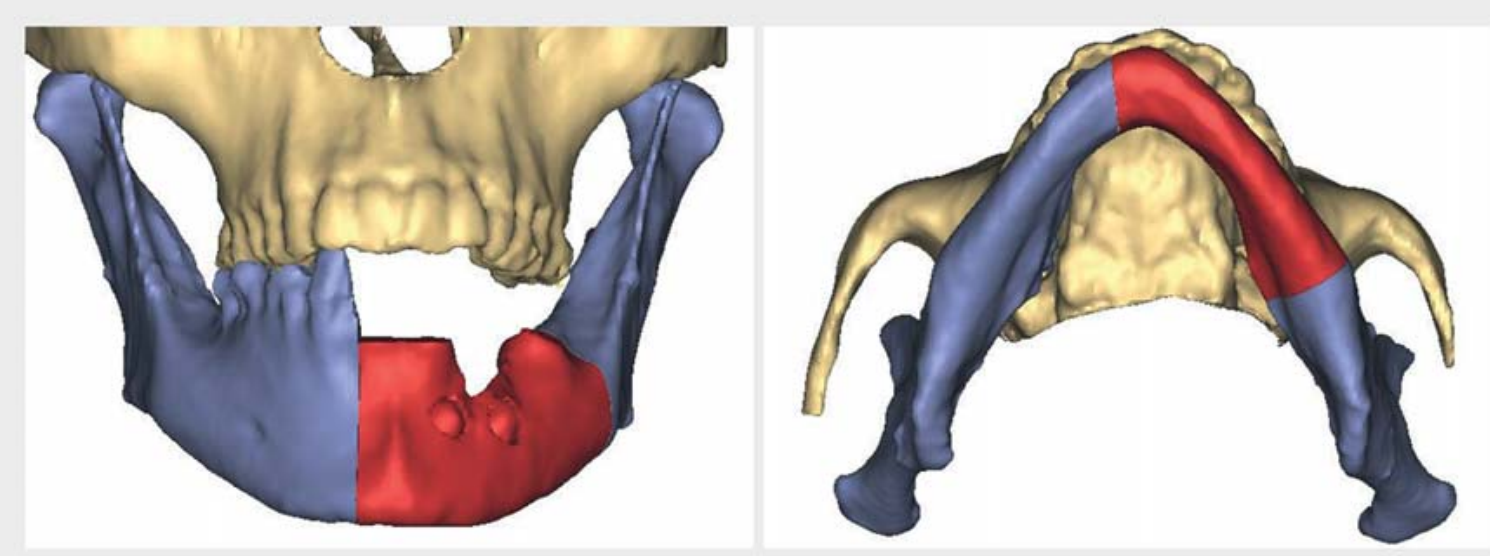

- Abb. 5 Präoperativer virtueller Plan der operativen Resektion des linken Unterkiefersegments bei einem Ameloblastom.

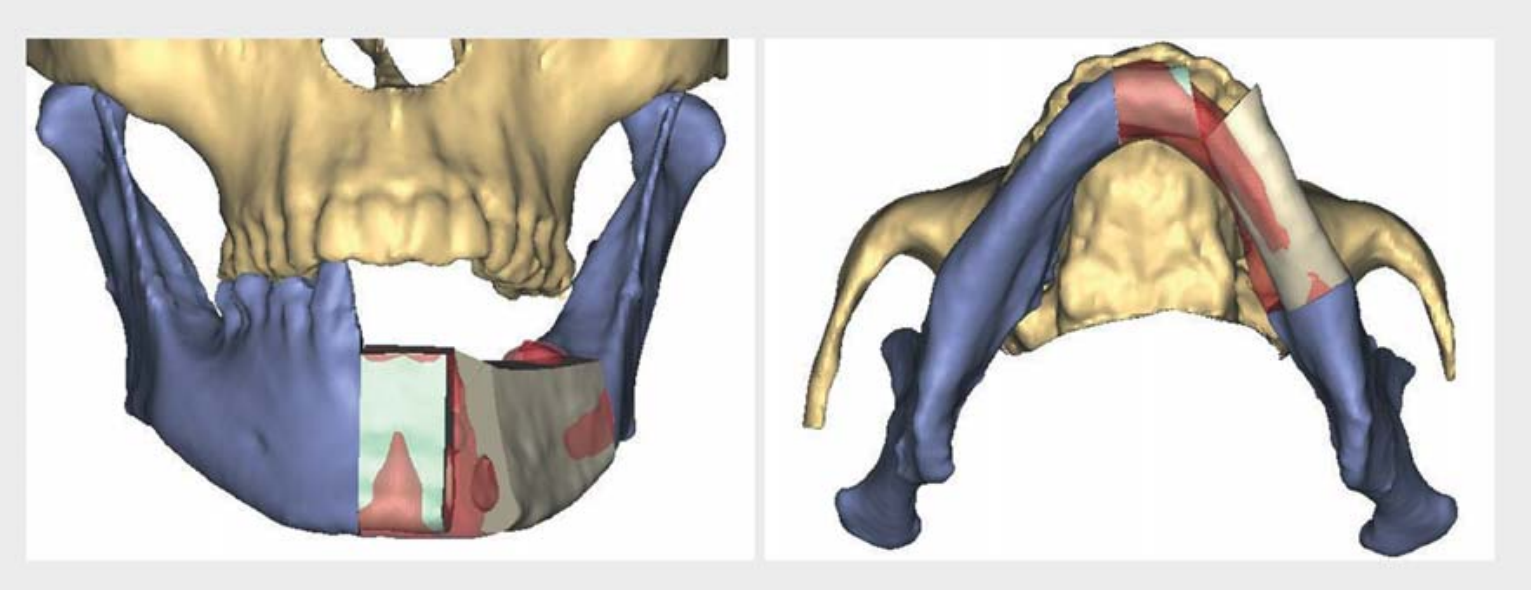

- Abb. 6 Virtuelle Planung der Unterkieferrekonstruktion mittels mikrovaskulärem Beckenkammtransplantat mit einer Osteotomie anterior.

dicus oder des N. glutealis sowie die Möglichkeit einer schweren Blutung [26]. In der Literatur zeigten sich jedoch im direkten Vergleich von anteriorer zu posteriorer Entnahmestelle deutlich geringere postoperative Komplikationen bei der Entnahme aus dem posterioren Beckenkamm [23, 30].

Die Knochenentnahme erfolgt in Bauchlage des Patienten. Die Zuhilfenahme eines großen Sandsackes oder mehrerer Tücher unter dem anterioren Beckenkamm erleichtert die Lagerung des Beckens und schafft respiratorischen Raum für die Beatmung durch den Anästhesisten. Es empfiehlt sich, die anatomischen Referenzpunkte, wie Wirbelsäule und Verlauf des posterioren Beckenkamms, vor der Inzision anzuzeichnen. Die Inzision verläuft ca. $2 \mathrm{~cm}$ lateral zum Beckenkamm und braucht eine Länge von $10 \mathrm{~cm}$ nicht zu überschreiten. Danach wird die gluteale Faszie scharf durchtrennt. Die Spina iliaca posterior superior stellt die anatomische Grenze der Inzision dar und schützt das Lig. sacroiliacum vor Beschädigungen $[26,28]$. Nach der subperiostalen Darstellung des Beckens kann die Knochenentnahme sowohl rein kortikal, kortikospongiös oder rein spongiös erfolgen.

\section{Mikrovaskuläres Beckenkamm- transplantat}

Besteht der Bedarf an großen Knochentransplantaten zur Rekonstruktion erheblicher Knochenanteile am Gesichtsschädel, so werden i.d.R. mikrovaskuläre Beckenkamm-, Fibula- und Skapulatransplantate herangezogen [8,31]. 


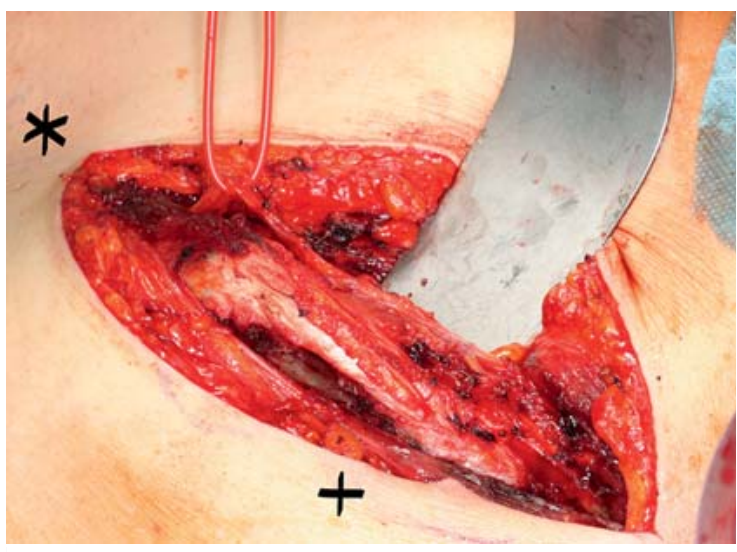

- Abb. 7 Mikrovaskuläres Beckenkammtransplantat links nach Präparation und Darstellung des Gefäßstiels ( ${ }^{*}$ anterior, + lateral).

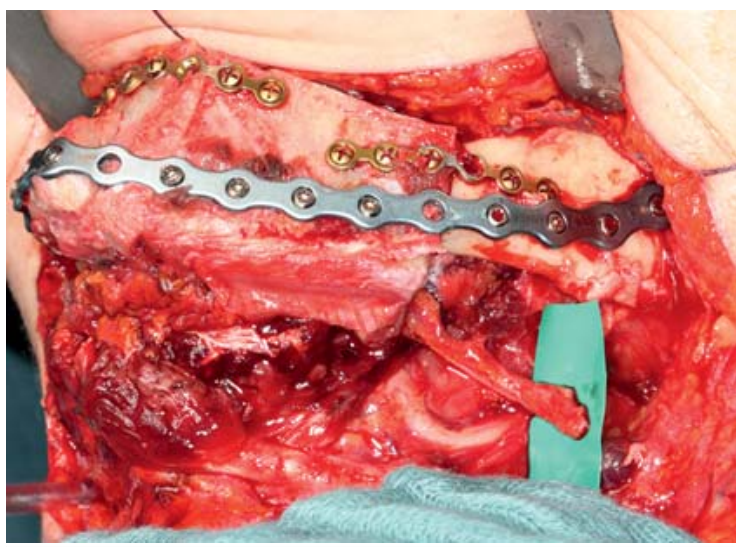

- Abb. 9 Mittels Osteosynthese fixiertes mikrovaskuläres Beckenkammtransplantat vor Anastomosierung.

Zu den häufigsten Ursachen solcher Defekte oder kompromittierter Empfängerlager zählen benigne und maligne Tumoren, Osteonekrosen, Osteomyelitiden sowie gravierende Traumata, wie z.B. Schussverletzungen. Durch die Verbesserung der Mikroskoptechnik gelang es in den 1960er- und 1970er-Jahren, kleinere Gefäße zu anastomosieren und somit großflächige knöcherne Strukturen durch mikrochirurgischen Gewebetransfer zu rekonstruieren [32]. Die Auswahl eines solchen knöchernen mikrochirurgischen Transplantats wird dabei wesentlich von der Größe, Lage, dem Weichgewebeangebot im Bereich des knöchernen Defizits sowie der Position und Qualität der vorhandenen Anschlussgefäße bestimmt. So steigt die Prognose einer knöchernen Rekonstruktion deutlich, wenn bei Kontinuitätsdefekten über $4 \mathrm{~cm}$ Länge ein mikrovaskuläres Transplantat verwendet wird [33]. Liegt ein bis zur Mitte reichender Unterkieferdefekt vor, der keinen Weichgewebsverlust aufweist, so stellt ein mikro-

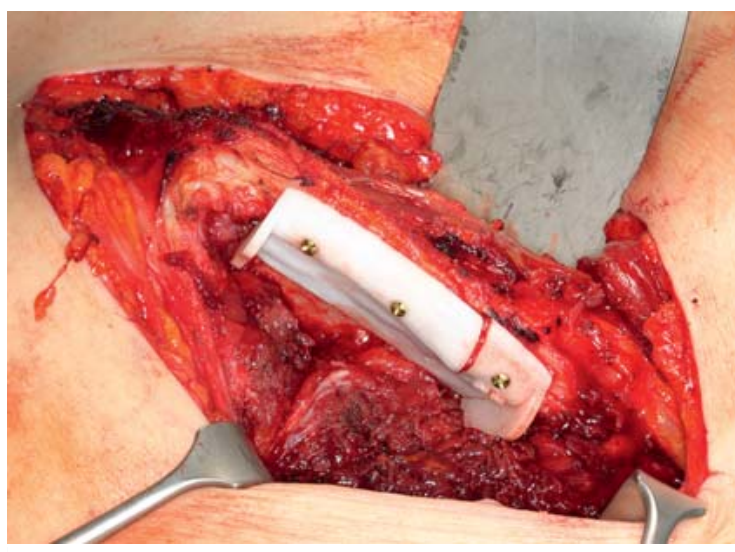

- Abb. 8 Anbringen der Osteotomieschablone an die virtuell geplante Resektionsstelle mit vorgegebenen Osteotomiehilfen und Schonung der Spina iliaca anterior superior.

vaskuläres Beckenkammtransplantat das Mittel der Wahl dar $[31,34,35]$.

Des Weiteren hat sich in der implantologischen Versorgung gezeigt, dass sich das mikrovaskuläre Fibulatransplantat, gefolgt vom mikrovaskulären Beckentransplantat, am widerstandsfähigsten gegenüber periimplantären Infektionen erweist und somit der Langzeiterfolg der prothetischen Rehabilitation steigt [36].

Liegt ein Defekt des Unterkiefers vor, der bis zur Hälfte reicht und kein weichgewebiges Defizit aufweist, so stellt das mikrovaskuläre Beckenkammtransplantat eine sehr gute Behandlungsoption dar [31]. Erstmals wurde es 1978 durch Taylor und Watson [37] sowie Sanders und Mayou [38] beschrieben.

Bedingt durch die Form des knöchernen Transplantats sowie der damit einhergehenden Knochenmenge eignet es sich hervorragend zur Unterkieferrekonstruktion und durch das Einbringen enossaler Implantate zur dentalen Rehabilitation. Es findet ebenfalls Einsatz im Bereich der aufwendigen Rekonstruktion von Maxilla [39] oder komplexen Mittelgesichtsdefekten [40].

Myoossäre Beckenkammtransplantate bestehen aus dem Knochen der Crista iliaca mit anhaftender Muskulatur, osteomuskulokutane Beckenkammtransplantate aus Beckenknochen, anhaftener Muskulatur sowie einem Hautanteil. Beide Transplantatformen können grundsätzlich sowohl an $\operatorname{der} A$. und V. circumflexa ilium superficialis als auch an $A$. und $V$. circumflexa ilium profunda gestielt umschnitten werden [8]. In Bezug auf die Blutversorgung des Knochens stellt die A. circumflexa ilium profunda das zuverlässigere Gefäß dar [37,38,41]. Durch die Mitversorgung der Hautanteile durch die A. circumflexa ilium profunda können zur Rekonstruktion die Weichgewebs- 
anteile mitverwendet werden [35,42-44]. Durch das Einbeziehen von Anteilen des M. obliquus internus zur Auskleidung von Weichgewebsdefiziten in der Mundhöhle beim myoossären Transplantat ist eine rasche Epithelialisierung zu erwarten $[43,44]$.

Problematisch wird allerdings die Blutversorgung des Hautareals bei osteomuskulokutanen Transplantaten diskutiert, die an der A. circumflexa ilium profunda gestielt sind. In ca. 20\% der Fälle reicht die Versorgung der Haut über das tiefe Gefäßsystem nicht aus [8]. Bei osteomuskulokutanen Transplantaten kann die venöse Drainage problematisch sein, wenn sie ausschließlich durch das tiefe Gefäßsystem erfolgt. Es wird aus diesem Grund empfohlen, immer die oberflächliche V. circumflexa ilium superficialis zusätzlich als Drainagegefäß einzusetzen [8]. Außerdem muss der relativ kurze Gefäßstiel bei der Wahl der zu anastomosierenden Anschlussgefäße bedacht werden [39].

Das Überleben eines mikrovaskulären Beckenkammtransplantats hängt signifikant von der Ischämiezeit ab. Besteht über einen längeren Zeitraum keine Perfusion, so steigt das Risiko des Implantatverlustes oder der Entstehung von Pseudarthrosen. Durch die Verwendung von computergestützten präoperativen Planungen mit Osteotomieschablonen kann jedoch die Ischämiezeit deutlich verringert werden, da die Anpassung des Transplantats in der Empfängerregion deutlich schneller erfolgt [45].

Durch die Weiterentwicklung der computergestützten Planung ist es inzwischen möglich, präoperativ die Implantation enossaler dentaler Implantate zu simulieren. Das Inserieren der dentalen Implantate ist mit präoperativ virtuell geplanten Positionsmarkierungen durch angefertigte Operationsschablonen bereits beim Heben des mikrovaskulären Beckenkammtransplantats möglich [46].

Das Volumen des entnommen Beckenkammtransplantats hat entscheidenden Einfluss auf die postoperative Donor-Site-Morbidität, wobei diese in der Literatur als moderat beschrieben wird [47]. Dennoch sollte immer berücksichtigt werden, dass die Entnahme von mikrovaskulärem Beckenkamm ebenfalls mit nicht unerheblichen Komplikationen wie Hernien, Frakturen oder Gangschwierigkeiten einhergehen kann. Wird eine Rekonstruktion des Kieferwinkels nicht benötigt, so besteht die Möglichkeit des Erhalts der Spina iliaca anterior superior, um somit die Donor-Site-Morbidität weiter zu reduzieren. Durch die Hilfe der virtuellen Planung bei der Rekonstruktion kann die entnommene Knochenmenge perfekt vorherbestimmt und damit auf ein Minimum reduziert werden [48].

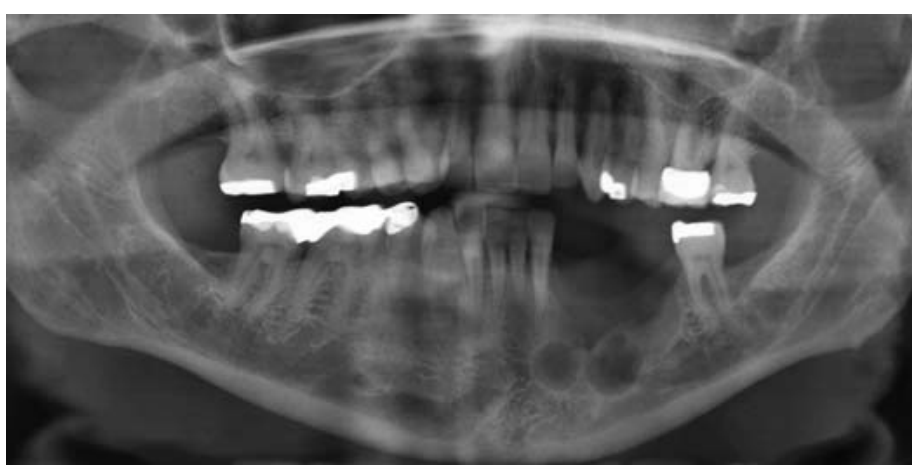

- Abb. 10 Präoperatives OPG. Histologisch gesichertes Ameloblastom im linken Unterkieferkorpus.

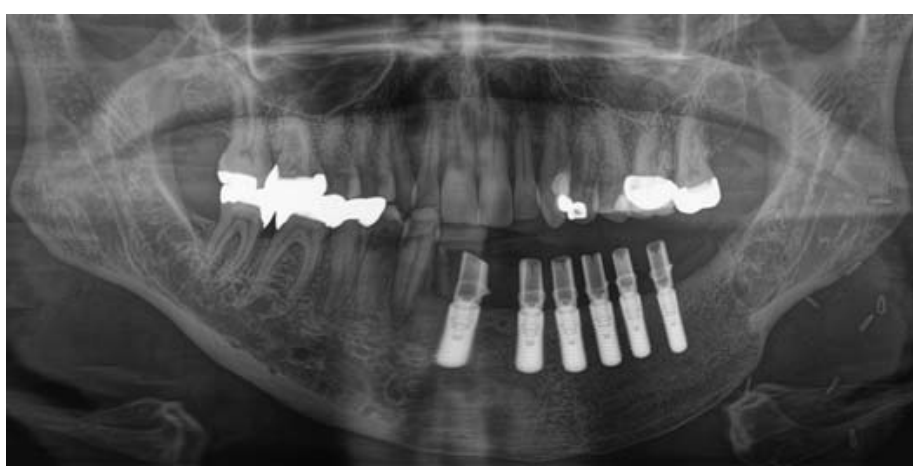

- Abb. 11 Postoperatives OPG nach implantatprothetischer Versorgung des mikrovaskulären Beckenkammtransplantats.

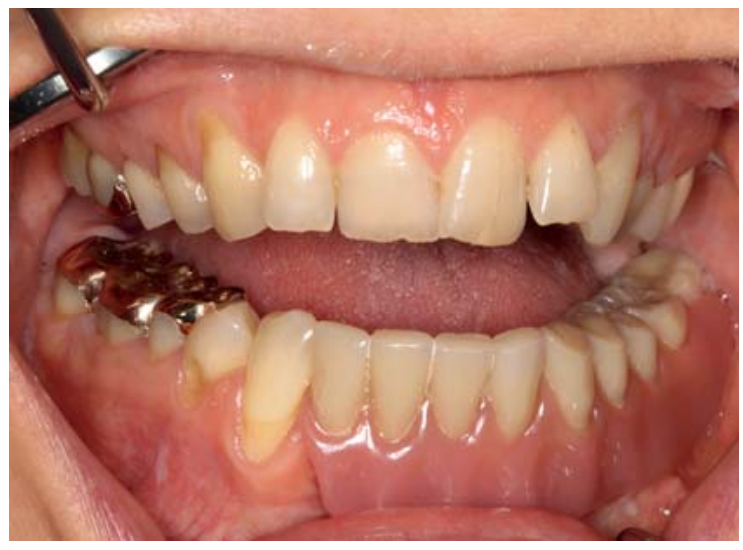

- Abb. 12 Intraorale Situation mit der eingesetzten implantatprothetischen Versorgung im linken Unterkiefer.

Interessenkonflikt

Die Autoren geben an, dass kein Interessenkonflikt besteht. 
Autorinnen/Autoren

\section{Dr. Dr. Nora Lautner}

Klinik und Poliklinik für Mund-, Kiefer- und Gesichtschirurgie, Universitätsklinikum RWTH Aachen

\section{Univ.-Prof. Dr. Dr. Frank Hölzle}

Klinik und Poliklinik für Mund-, Kiefer- und Gesichtschirurgie, Universitätsklinikum RWTH Aachen

\section{PD Dr. Dr. Ali Modabber}

Klinik und Poliklinik für Mund-, Kiefer- und Gesichtschirurgie, Universitätsklinikum RWTH Aachen

\section{Korrespondenzadresse}

Univ.-Prof. Dr. Dr. Frank Hölzle

Klinik und Poliklinik für Mund-, Kiefer- und Gesichtschirurgie Universitätsklinikum RWTH Aachen

Pauwelsstraße 30

52074 Aachen

fhoelzle@ukaachen.de

\section{Literatur}

[1] Albee FH, Weigel EP. Restoration of loss of bone: including an analysis of the first hundred cases of fracture treated by bone graft at US Army General Hospital No. 3, Colonia, NJ. J Am Med Assoc 1920; 74: 589-597

[2] Mowlem AR. Cancellous chip grafts for the restoration of bone defects. Lancet 1945; ii: 746

[3] Blocker T jr., Weiss L. Use of cancellous bone in the repair of defects about the jaws. Ann Surg 1946; 123 : 622

[4] Dimitriou R, Mataliotakis Gl, Angoules AG et al. Complications following autologous bone graft harvesting from the iliac crest and using the RIA: a systematic review. Injury 2011; 42 (Suppl. 2): S3-S15

[5] Kummoona R. Chondro-osseous iliac crest graft for one stage reconstruction of the ankylosed TM] in children. J Maxillofac Surg 1986; 14: 215-220

[6] Maclntosh RB, Henny FA. A spectrum of application of autogenous costochondral grafts. J Maxillofac Surg 1977; 5: 257267

[7] Wolford LM, Cottrell DA, Henry C. Sternoclavicular grafts for temporomandibular joint reconstruction. J Oral Maxillofac Surg 1994; 52: 119-128

[8] Hausamen JE, Machtens E, Reuther J, Eufinger $H$, Kübler A, Schliephake H. Mund-, Kiefer- und Gesichtschirurgie. Berlin, Heidelberg: Springer; 2012

[9] Baqain ZH, Anabtawi M, Karaky AA et al. Morbidity from anterior iliac crest bone harvesting for secondary alveolar bone grafting: an outcome assessment study. J Oral Maxillofac Surg 2009; 67; 570-575

[10] Thorwarth M, Srour S, Felszeghy E et al. Stability of autogenous bone grafts after sinus lift procedures: a comparative study between anterior and posterior aspects of the iliac crest and an intraoral donor site. Oral Surg Oral Med Oral Pathol Oral Radiol Endod 2005; 100: 278-284

[11] Burk T, Del Valle J, Finn RA et al. Maximum quantity of bone available for harvest from the anterior iliac crest, posterior iliac crest, and proximal tibia using a standardized surgical ap- proach: a cadaveric study. I Oral Maxillofac Surg 2016; 74: 2532-2548

[12] Schaaf H Lendeckel S, Howaldt H-P et al. Donor site morbidity after bone harvesting from the anterior iliac crest. Oral Surg Oral Med Oral Pathol Oral Radiol Endod 2010; 109: 52-58

[13] Swan M, Goodacre T. Morbidity at the iliac crest donor site following bone grafting of the cleft alveolus. $\mathrm{Br}$ J Oral Maxillofac Surg 2006; 44: 129-133

[14] Fretwurst T, Wanner L, Nahles $S$ et al. A prospective study of factors influencing morbidity after iliac crest harvesting for oral onlay grafting. J Craniomaxillofac Surg 2015; 43: 705-709

[15] Scott W, Petersen RC, Grant S. A method of procuring iliac bone by trephine curettage. J Bone Joint Surg Am 1949; 31 : 860

[16] Freilich MM, Sándor GK. Ambulatory in-office anterior iliac crest bone harvesting. Oral Surg Oral Med Oral Pathol Oral Radiol Endod 2006; 101: 291-298

[17] Mrazik J, Amato C, Leban S et al. The ilium as a source of au togenous bone for grafting: clinical considerations. J Oral Surg Am Dent Assoc 1965 1980; 38: 29-32

[18] Farhood V, Ryan D, Johnson R. A modified approach to the ilium to obtain graft material. J Oral Surg Am Dent Assoc 1965 1978; 36: 784-786

[19] Kazanjian VH, Converse JM. Kazanjian \& Converse's surgical treatment of facial injuries (Vol. 1). Baltimore: Williams \& Wilkins; 1974: 573-577

[20] Levy R, Siffert R. Inner table iliac bone graft. Surg Gynecol Obstet 1969; 128: 605

[21] Hall M, Smith R. The medial approach for obtaining iliac bone. J Oral Surg Am Dent Assoc 1965 1981; 39: 462-465

[22] Hall MB, Vallerand WP, Thompson D et al. Comparative anatomic study of anterior and posterior iliac crests as donor sites. J Oral Maxillofac Surg 1991; 49: 560-563

[23] Ahlmann E, Patzakis M, Roidis $\mathrm{N}$ et al. Comparison of anterior and posterior iliac crest bone grafts in terms of harvest-site morbidity and functional outcomes. J Bone Joint Surg Am 2002; 84: 716-720

[24] Ebraheim N A, Yang H, Lu J et al. Anterior iliac crest bone graft: anatomic considerations. Spine 1997; 22: 847-849

[25] Kurz LT, Garfin SR, Booth RE jr. Harvesting autogenous iliac bone grafts: a review of complications and techniques. Spine 1989: 14: 1324-1331

[26] Bloomquist DS, Feldman GR. The posterior ilium as a donor site for maxillo-facial bone grafting. J Maxillofac Surg 1980; 8: 60-64

[27] Engelstad ME, Morse T. Anterior iliac crest, posterior iliac crest and proximal tibia donor sites: a comparison of cancellous bone volumes in fresh cadavers. J Oral Maxillofac Surg 2010; 68: 3015-3021

[28] Sittitavornwong S, Falconer DS, Shah R et al. Anatomic considerations for posterior iliac crest bone procurement. J Oral Maxillofac Surg 2013; 71: 1777-1788

[29] Dingman RO. The use of iliac bone in the repair of facial and cranial defects. Plast Reconstr Surg 1950; 6: 179-195

[30] Kolomvos N, latrou I, Theologie-Lygidakis $\mathrm{N}$ et al. Iliac crest morbidity following maxillofacial bone grafting in children: a clinical and radiographic prospective study. J Craniomaxillofac Surg 2010; 38: 293-302

[31] Modabber A, Peters F, Raith S et al. Virtuelle Planung komplexer mikrovaskulärer knöcherner Rekonstruktionen in der MKG-Chirurgie. MKG-Chir 2017; 10: 272-283 
[32] Ueba Y, Fujikawa S. Nine years' follow-up of a free vascularized fibular graft in neurofibromatosis: a case report and literature review. Jpn J Orthop Trauma Surg 1983; 26: 595-600

[33] Kärcher H, Penkner K. Ergebnisse der freien und gefäßgestielten Knochenrekonstruktion nach Unterkieferkontinuitätsdefekten. Dtsch Z Mund Kiefer Gesichtschir 1991; 15: 285-291

[34] Bitter K. Bone transplants from the iliac crest to the maxillofacial region by the microsurgical technique. J Maxillofac Surg 1980; 8: 210-216

[35] Riediger D. Restoration of masticatory function by microsurgically revascularized iliac crest bone grafts using enosseous implants. Plast Reconstr Surg 1988; 81: 861-876

[36] Blake F, Bubenheim M, Heiland $M$ et al. Retrospective assessment of the peri-implant mucosa of implants inserted in reanastomosed or free bone grafts from the fibula or iliac crest. Int J Oral Maxillofac Implants 2008; 23: 1102-1108

[37] Taylor GI, Daniel RK. The anatomy of several free flap donor sites. Plast Reconstr Surg 1975; 56: 243-253

[38] Sanders R, Mayou B]. A new vascularized bone graft transferred by microvascular anastomosis as a free flap. $\mathrm{Br}$ । Surg 1979; 66: 787-788

[39] Bianchi B, Ferri A, Ferrani $S$ et al. lliac crest free flap for maxillary reconstruction. J Oral Maxillofac Surg 2010; 68: 27062713

[40] Modabber A, Gerresen M, Ayoub N et al. Computer-assisted zygoma reconstruction with vascularized iliac crest bone graft. Int J Med Robot 2013; 9: 497-502

[41] Taylor GI, Watson N. One-stage repair of compound leg defects with free, revascularized flaps of groin skin and iliac bone. Plast Reconstr Surg 1978; 61: 494-506

[42] Kärcher H. Die Unterkieferrekonstruktion mit freien mikrovaskulären Knochentransplantaten. Acta Chir Austriaca 1986; 33: 251
[43] Urken ML, Vickery C, Weinberg $\mathrm{H}$ et al. The internal obliqueiliac crest osseomyocutaneous microvascular free flap in head and neck reconstruction. J Reconstr Microsurg 1989; 5: 203214

[44] Urken ML, Vickery C, Weinberg $\mathrm{H}$ et al. The internal obliqueiliac crest osseomyocutaneous free flap in oromandibular reconstruction: report of 20 cases. Arch Otolaryngol Neck Surg 1989; 115: 339-349

[45] Modabber A, Legros C, Rana M et al. Evaluation of computerassisted jaw reconstruction with free vascularized fibular flap compared to conventional surgery: a clinical pilot study. Int J Med Robot 2012; 8: 215-220

[46] Modabber A, Möhlhenrich SC, Ayoub N et al. Computer-aided mandibular reconstruction with vascularized iliac crest bone flap and simultaneous implant surgery. J Oral Implantol 2015; 41: e189-e194

[47] Ghassemi A, Ghassemi M, Riediger D et al. Comparison of donor-site engraftment after harvesting vascularized and nonvascularized iliac bone grafts. J Oral Maxillofac Surg 2009; 67: 1589-1594

[48] Ayoub N, Ghassemi A, Rana M et al. Evaluation of computerassisted mandibular reconstruction with vascularized iliac crest bone graft compared to conventional surgery: a randomized prospective clinical trial. Trials 2014; 15: 114

\section{Bibliografie}

DOI https://doi.org/10.1055/s-0043-123614

OP-JOURNAL 2018; 34: 61-67 @ Georg Thieme Verlag KC Stuttgart · New York ISSN 0178-1715 